\title{
Cost Analysis of Activities for Network Drug Information Centers at the Ministry of Health Hospitals in Saudi Arabia
}

\author{
Yousef Ahmed Alomi ${ }^{1, *}$ (D), Ban Abdullah Aldosary ${ }^{2}$ \\ ${ }^{I}$ The Former General Manager of General Administration of Pharmaceutical Care, The Past Head, National Clinical \\ Pharmacy and Pharmacy Practice, The Past Head, Pharmacy R and D Administration, Ministry of Health, Riyadh, \\ SAUDI ARABIA. \\ ${ }^{2}$ Pharmaceutical Care Department, Al-Amel Mental City, Ministry of Health, Riyadh, SAUDI ARABIA.
}

\section{Abstract}

Objectives: In this study, we aimed to analyze the cost of network drug information services at the Ministry of Health $(\mathrm{MOH})$ hospitals in Saudi Arabia. Method: In this study, we simulated the 2-month cross-sectional survey data of all drug information centers at the $\mathrm{MOH}$ hospitals. Any drug store that has provided services to the healthcare professionals and the public participated in the questionnaire. National, regional and local drug information centers in the healthcare institutions participated in this survey. All type of hospitals or primary care centers (e.g. public, pediatric, maternity and psychiatry) were included in this study. The survey consisted of two parts: the first part collects demographics data and the second part analyzes the cost of activities of drug information centers. The clinical activities were derived from the model of the American College of Clinical Pharmacy (ACCP). The type cost consisted of related central drug information activities, the cost of the patient-centered drug information activity and the cost of Administrative drug information activities. All cost used US dollar currency. Results: The survey was distributed to 60 drug information centers and a total of 46 centers responded to the survey; the response rate was $76.66 \%$. Most of the hospitals (11 (23.9\%)) had $100-199$ beds and the others $(11(23.9 \%))$ had $200-299$ beds. The total daily cost of drug information activities was $6,002.18$ USD. The highest cost of drug information activities was central drug information activities $(2,654.69$ USD (44.23\%)) followed by the administration of drug information activities (2,280.59 USD (37.99\%)) and patient-specific drug information activities (1,066.9 USD (17.77\%)). The highest daily cost of drug information activities among central drug information center was responding to drug information inquiries ((536.24 USD)) followed by the residential training ((9274.46 USD)) and pharmacist's training ((263.14 USD)). Conclusion: The central activities of drug information centers costed the highest. Expanding the services to patient-specific activities will decrease morbidity, mortality and justify cost analysis at $\mathrm{MOH}$ hospitals in Saudi Arabia.

Key words: Cost Analysis, Network, Drug Information Centers, Activities, Ministry of Health, Saudi Arabia.
\end{abstract}

Received: 13-09-2018;

Accepted: 17-11-2018

*Correspondence to:

Dr. Yousef Ahmed Alomi

The Former General Manager of General Administration of Pharmaceutical Care, The Former Head, National Clinical Pharmacy and Pharmacy Practice, The Past Head, Pharmacy R and D Administration, Ministry of Health, Riyadh, SAUDI ARABIA.

Phone no: +966 504417712 Email: yalomi@gmail.com

DOI: 10.5530/ijpcs.2019.8.9

Copyright: ๑ the author(s),publisher and licensee International Journal of Pharmacology and Clinical Sciences. This is an open-access article distributed under the terms of the Creative Commons Attribution Non-Commercial License, which permits unrestricted noncommercial use, distribution, and reproduction in any medium, provided the original work is properly cited.

This is an open access article distributed under the terms of the Creative Commons Attribution-NonCommercial-ShareAlike 4.0 License

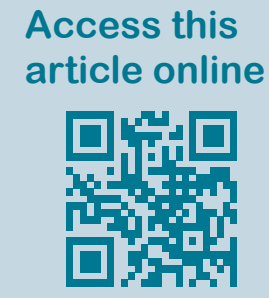

www.ijpcs.net

\section{INTRODUCTION}

The Drug information centers (DICs) provide several activities and services to healthcare professionals and patients. The American Society of Health-System Pharmacists explored the functions of drug information services. ${ }^{[1]}$ The DICs services are provided to the inside or outside of healthcare organization. The DICs may contract with public or private institutions for outsourcing their services. All services are needed for the quantification of the cost services. Several investigations have been published regarding the cost avoidance of 
drug information centers. ${ }^{[2-8]}$ However, to the best of our knowledge, there are no studies regarding the cost of drug information services in overseas countries or in local Middle Eastern countries. Therefore, in this study, we aimed to explore the cost analysis of drug information services in the Kingdom of Saudi Arabia (KSA).

\section{METHODS}

In this study, we simulated the 2-month cross-sectional survey data of all the drug information centers at the Ministry of Health hospitals, Saudi Arabia. Any drug store that has provided services to the healthcare professionals and the public participated in the questionnaire. All types of drug information centers (national, regional or local) were included in the survey. All type of hospitals and primary care centers were included in the survey (public, pediatric, maternity and psychiatry). The survey consisted of two parts: the first part collects demographic data and the second part consists of a questionnaire on four domains. (1) the first domain is regarding workload of drug information centers; (2) the second domain is regarding cost analysis of drug information centers; (3) the third domain is related to cost analysis of drug information activities; and (4) the fourth domain is about education and training activity of drug information centers. This article discusses the third domain. It includes the clinical activities derived from the model of the American College of Clinical Pharmacy (ACCP), ${ }^{[9]}$ which includes the costs of central drug information activities, patientcentered drug information activities and administrative drug information activities. All costs were calculated in US dollar currency. All analysis were conducted in an electronic Survey Monkey system.

\section{RESULTS}

The survey was distributed to 60 drug information centers among which 46 responded; the response rate was $76.66 \%$. Most of the hospitals (11 (23.9\%)) had 100-199 beds and the others (11 (23.9\%)) had 200-299 beds. Most of the hospitals had accreditation from the Central Board of Accreditation for Healthcare Institutions, Saudi Arabia (CBAHI) (27 (58.7\%)) and the Saudi Commission of Health Specialties (9 (19.6\%)), whereas $11(23.9 \%)$ hospitals were not accredited by any organization. Most of the drug information centers were for adults $(20(43.48 \%))$ and general specialties $15(32.61 \%)$ followed by psychiatric drug information centers $(5(10.87 \%))$ and pediatric drug

\begin{tabular}{|c|c|c|}
\hline Number of beds at the hospital & $\begin{array}{l}\text { Response } \\
\text { Count }\end{array}$ & $\begin{array}{l}\text { Response } \\
\text { Percent }\end{array}$ \\
\hline$<50$ & 7 & $15.2 \%$ \\
\hline $50-99$ & 6 & $13.0 \%$ \\
\hline 100-199 & 11 & $23.9 \%$ \\
\hline 200-299 & 11 & $23.9 \%$ \\
\hline $300-399$ & 4 & $8.7 \%$ \\
\hline $400-499$ & 4 & $8.7 \%$ \\
\hline$=$ or $>600$ & 3 & $6.5 \%$ \\
\hline Medical City & 0 & $0.0 \%$ \\
\hline Answered question & 46 & \\
\hline Skipped question & 0 & \\
\hline The hospital accreditation & $\begin{array}{l}\text { Response } \\
\text { Count }\end{array}$ & $\begin{array}{l}\text { Response } \\
\text { Percent }\end{array}$ \\
\hline CIBAHI & 27 & $58.7 \%$ \\
\hline Joint Commotion the USA & 7 & $15.2 \%$ \\
\hline Canada & 1 & $2.2 \%$ \\
\hline Saudi commission on health accreditation & 9 & $19.6 \%$ \\
\hline Non accredited & 11 & $23.9 \%$ \\
\hline Answered question & 46 & \\
\hline Skipped question & 0 & \\
\hline $\begin{array}{l}\text { The type of drug information } \\
\text { center }\end{array}$ & $\begin{array}{l}\text { Response } \\
\text { Count }\end{array}$ & $\begin{array}{l}\text { Response } \\
\text { Percent }\end{array}$ \\
\hline General Drug Information Center & 15 & $32.61 \%$ \\
\hline Adult drug information center & 20 & $43.48 \%$ \\
\hline Pediatric drug information center & 4 & $8.70 \%$ \\
\hline Psychiatric drug information center & 5 & $10.87 \%$ \\
\hline Oncology drug information center & 0 & $0.00 \%$ \\
\hline Cardiology drug information center & 1 & $2.17 \%$ \\
\hline Dental drug information center & 0 & $0.00 \%$ \\
\hline Primary health care drug information center & 1 & $2.17 \%$ \\
\hline Answered question & 46 & \\
\hline Skipped question & 0 & \\
\hline
\end{tabular}

information centers (4 (8.7\%)) (Table 1). Among the responders, 43 (95.6\%) were Saudi and 2 (4.4\%) were non-Saudi nationals. The patients belonged to the age group of 18-40 years (44 (95.7\%)). The highest level of education of the responders was Bachelor of Pharmacy (22 (47.83\%)), Doctor of Pharmacy (11 (23.9\%)) and Master of Science $(9(19.57 \%))$. Only $4(10 \%)$ responders were certified in the field of pharmaceutical specialties. Most of the responders (30 (65.2\%)) had an experience of 1-6 years with drug information services, whereas $8(17.39 \%)$ responders had clinical pharmacy experience (Tables 2 and 3). The highest average salary of the head of the drug information pharmacist was 143.68 USD per day, followed by the regular pharmacist (132.26 USD) and clinical pharmacist (128.34 USD) 


\begin{tabular}{|c|c|c|}
\hline Nationality & $\begin{array}{l}\text { Response } \\
\text { Count }\end{array}$ & $\begin{array}{l}\text { Response } \\
\text { Percent }\end{array}$ \\
\hline Saudi & 43 & $95.6 \%$ \\
\hline Non- Saudi & 2 & $4.4 \%$ \\
\hline Answered question & 45 & \\
\hline Skipped question & 1 & \\
\hline Age & $\begin{array}{l}\text { Response } \\
\text { Count }\end{array}$ & $\begin{array}{l}\text { Response } \\
\text { Percent }\end{array}$ \\
\hline $18-40$ years & 44 & $95.7 \%$ \\
\hline $40-65$ years & 2 & $4.3 \%$ \\
\hline 18- 40 years & 0 & $0.0 \%$ \\
\hline more than 65 years & 0 & $0.0 \%$ \\
\hline Answered question & 46 & \\
\hline Skipped question & 0 & \\
\hline Academic Qualification (s): & $\begin{array}{l}\text { Response } \\
\text { Count }\end{array}$ & $\begin{array}{l}\text { Response } \\
\text { Percent }\end{array}$ \\
\hline Diploma Pharmacy & 7 & $15.22 \%$ \\
\hline Bsc. Pharm & 22 & $47.83 \%$ \\
\hline M.S & 9 & $19.57 \%$ \\
\hline Msc. Clinical Pharmacy & 6 & $13.04 \%$ \\
\hline Pharm.D. & 11 & $23.91 \%$ \\
\hline Ph.D & 0 & $0.00 \%$ \\
\hline MBA & 3 & $6.52 \%$ \\
\hline Pharmacy Residency Two years (R1) & 1 & $2.17 \%$ \\
\hline Pharmacy Residency one year (R2) & 0 & $0.00 \%$ \\
\hline Fellowship & 1 & $2.17 \%$ \\
\hline Others & 1 & $2.17 \%$ \\
\hline Answered question & 46 & \\
\hline Skipped question & 0 & \\
\hline $\begin{array}{l}\text { Total years worked as a } \\
\text { pharmacist }\end{array}$ & $\begin{array}{l}\text { Response } \\
\text { Count }\end{array}$ & $\begin{array}{l}\text { Response } \\
\text { Percent }\end{array}$ \\
\hline $\begin{array}{l}\text { Board Certified Ambulatory Care } \\
\text { Pharmacist (BCACP) }\end{array}$ & 0 & $0.0 \%$ \\
\hline $\begin{array}{l}\text { Board Certified Critical Care } \\
\text { Pharmacist (BCCCP) }\end{array}$ & 0 & $0.0 \%$ \\
\hline $\begin{array}{l}\text { Board Certified Nuclear Pharmacist } \\
\text { (BCNP) }\end{array}$ & 1 & $2.5 \%$ \\
\hline $\begin{array}{l}\text { Board Certified Nutrition Support } \\
\text { Pharmacist (BCNSP) }\end{array}$ & 0 & $0.0 \%$ \\
\hline $\begin{array}{l}\text { Board Certified Oncology Pharmacist } \\
\text { (BCOP) }\end{array}$ & 0 & $0.0 \%$ \\
\hline $\begin{array}{l}\text { Board Certified Pediatric Pharmacy } \\
\text { Specialist (BCPPS) }\end{array}$ & 1 & $2.5 \%$ \\
\hline $\begin{array}{l}\text { Board Certified Pharmacotherapy } \\
\text { Specialists (BCPS) }\end{array}$ & 1 & $2.5 \%$ \\
\hline $\begin{array}{l}\text { Board Certified Psychiatric } \\
\text { Pharmacist (BCPP) }\end{array}$ & 1 & $2.5 \%$ \\
\hline Non & 39 & $97.5 \%$ \\
\hline Others & 1 & $2.5 \%$ \\
\hline Answered question & 40 & \\
\hline Skipped question & 6 & \\
\hline
\end{tabular}

\begin{tabular}{|c|c|c|c|c|c|}
\hline 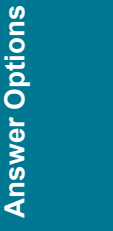 & 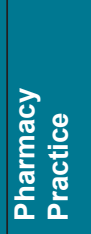 & 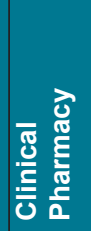 & 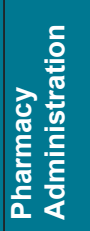 & 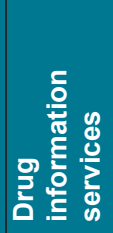 & 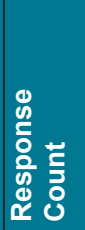 \\
\hline 0 & 2 & 7 & 3 & 3 & 13 \\
\hline$<1$ year & 3 & 5 & 4 & 6 & 16 \\
\hline $1-3$ & 8 & 5 & 9 & 14 & 24 \\
\hline $4-6$ & 17 & 3 & 7 & 16 & 28 \\
\hline$>6$ years & 23 & 5 & 11 & 8 & 29 \\
\hline \multicolumn{5}{|c|}{ answered question } & 46 \\
\hline \multicolumn{5}{|c|}{ skipped question } & 0 \\
\hline
\end{tabular}

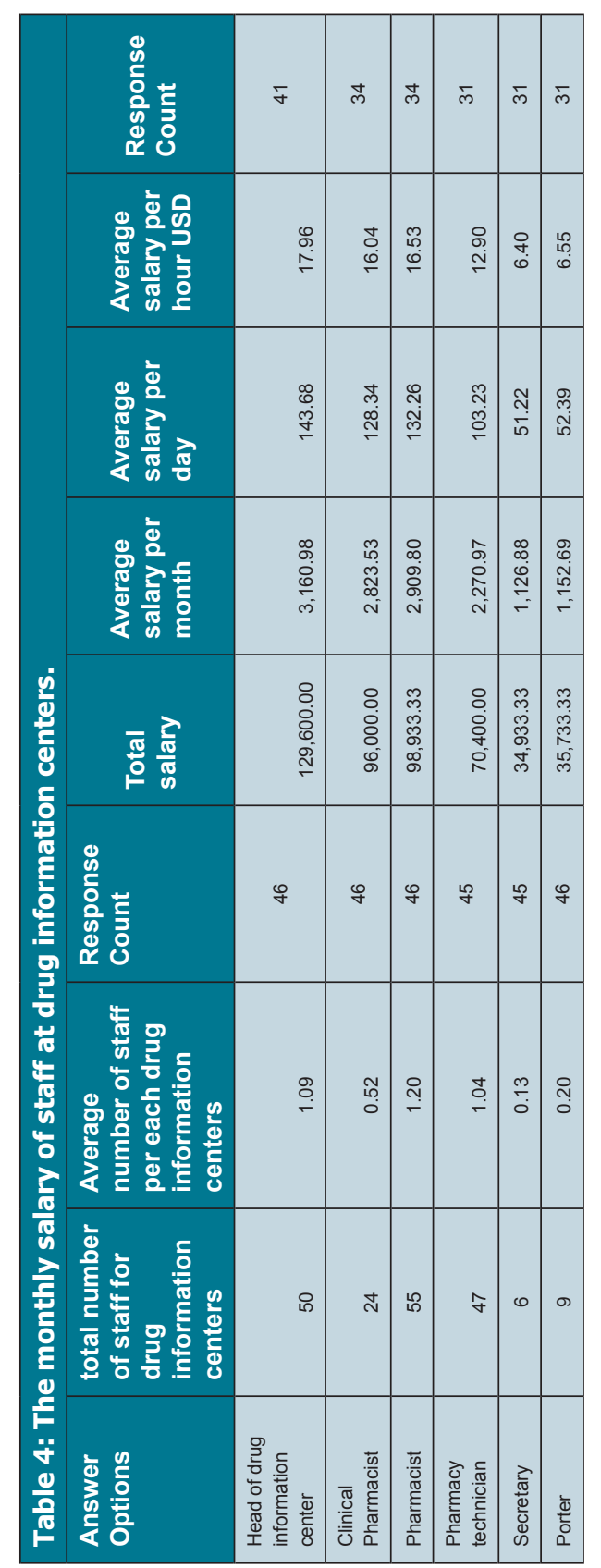




\begin{tabular}{|c|c|c|c|c|c|c|c|c|c|c|c|c|c|}
\hline Type of activities & 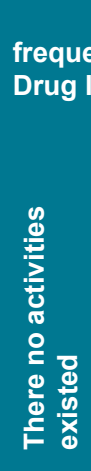 & 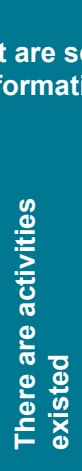 & 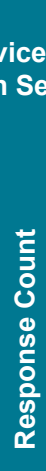 & 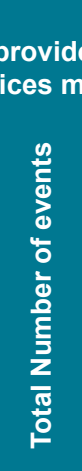 & 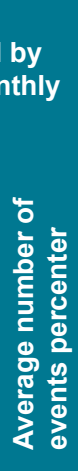 & 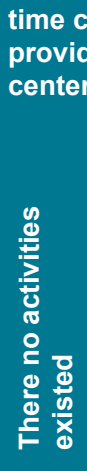 & 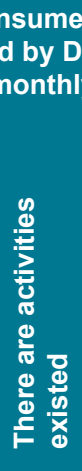 & 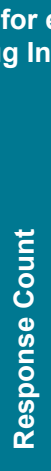 & 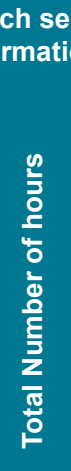 & 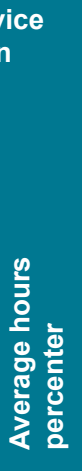 & 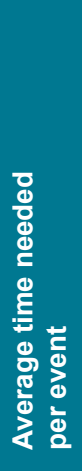 & 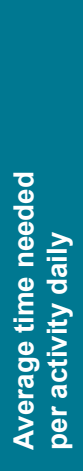 & 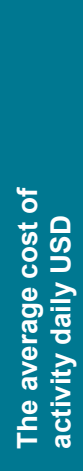 \\
\hline \multicolumn{14}{|c|}{ Cost of Central Drug Information Activities } \\
\hline \multicolumn{14}{|l|}{ Pharmacoeconomic Services } \\
\hline Drug Utilization Evaluation (DUE) & 21 & 24 & 45 & 152.5 & 6.35 & 19 & 23 & 42 & 39.63 & 1.72 & 10.95 & 1.37 & 173.53 \\
\hline $\begin{array}{l}\text { Cost reduction project } \\
\text { (Pharmacoeconomics) }\end{array}$ & 34 & 9 & 43 & 32.5 & 3.61 & 30 & 12 & 42 & 20.08 & 1.67 & 6.04 & 0.76 & 95.78 \\
\hline \multicolumn{14}{|l|}{ In-Services Education } \\
\hline $\begin{array}{l}\text { Deliver seminar and Presentation } \\
\text { or Lecture }\end{array}$ & 16 & 30 & 46 & 135 & 4.50 & 12 & 31 & 43 & 38.96 & 1.26 & 5.66 & 0.71 & 89.64 \\
\hline \multicolumn{14}{|l|}{ In-Services Training } \\
\hline Residency Training & 34 & 8 & 42 & 70 & 8.75 & 32 & 10 & 42 & 19.79 & 1.98 & 17.32 & 2.16 & 274.46 \\
\hline Pharmacist Training & 13 & 30 & 43 & 245 & 8.17 & 19 & 24 & 43 & 48.79 & 2.03 & 16.60 & 2.08 & 263.14 \\
\hline Pharmacy technician training & 20 & 24 & 44 & 145 & 6.04 & 19 & 24 & 43 & 44.46 & 1.85 & 11.19 & 1.40 & 177.40 \\
\hline Pharmacist Student Training & 20 & 24 & 44 & 210 & 8.75 & 18 & 25 & 43 & 47.13 & 1.89 & 16.50 & 2.06 & 261.45 \\
\hline Pharm D student training & 20 & 23 & 43 & 177.5 & 7.72 & 17 & 26 & 43 & 53 & 2.04 & 15.73 & 1.97 & 249.35 \\
\hline Pharmacy technician student training & 23 & 20 & 43 & 120 & 6.00 & 22 & 21 & 43 & 35.71 & 1.70 & 10.20 & 1.28 & 161.72 \\
\hline \multicolumn{14}{|l|}{ Drug Information Services } \\
\hline $\begin{array}{l}\text { Respond to Drug Information } \\
\text { Questions }\end{array}$ & 5 & 40 & 45 & 1072.5 & 26.81 & 5 & 39 & 44 & 49.21 & 1.26 & 33.83 & 4.23 & 536.24 \\
\hline \multicolumn{14}{|l|}{ Poisoning Information Services } \\
\hline Provider of Poison Information & 15 & 29 & 44 & 117.5 & 4.05 & 14 & 28 & 42 & 12.71 & 0.45 & 1.84 & 0.23 & 29.15 \\
\hline \multicolumn{14}{|l|}{$\begin{array}{l}\text { Clinical Researches and } \\
\text { Publication }\end{array}$} \\
\hline $\begin{array}{l}\text { Perform clinical research, publishing } \\
\text { articles }\end{array}$ & 31 & 14 & 45 & 50 & 3.57 & 30 & 13 & 43 & 30.04 & 2.31 & 8.25 & 1.03 & 130.81 \\
\hline Prepare and Publish Newsletter & 24 & 20 & 44 & 120 & 6.00 & 24 & 18 & 42 & 40.13 & 2.23 & 13.38 & 1.67 & 212.02 \\
\hline \multicolumn{14}{|c|}{ Cost of Patient-Specific Drug Information Activities } \\
\hline ADR (Identification \& Reporting) & 10 & 36 & 46 & 312.5 & 8.68 & 11 & 33 & 44 & 18.75 & 0.57 & 4.93 & 0.62 & 78.17 \\
\hline $\begin{array}{l}\text { Medications Errors preventing \& } \\
\text { monitoring }\end{array}$ & 5 & 41 & 46 & 605 & 14.76 & 5 & 38 & 43 & 33.75 & 0.89 & 13.11 & 1.64 & 207.73 \\
\hline Ambulatory care clinic participation & 22 & 21 & 43 & 127.5 & 6.07 & 18 & 24 & 42 & 26.67 & 1.11 & 6.75 & 0.84 & 106.94 \\
\hline CPR team participation & 24 & 19 & 43 & 72.5 & 3.82 & 22 & 21 & 43 & 18.46 & 0.88 & 3.35 & 0.42 & 53.17 \\
\hline Pharmacokinetic consultation & 19 & 24 & 43 & 150 & 6.25 & 18 & 24 & 42 & 23.5 & 0.98 & 6.12 & 0.76 & 97.00 \\
\hline Nutrition Support & 29 & 14 & 43 & 85 & 6.07 & 27 & 15 & 42 & 13.92 & 0.93 & 5.63 & 0.70 & 89.30 \\
\hline Patient Counseling & 10 & 33 & 43 & 607.5 & 18.41 & 7 & 35 & 42 & 52.13 & 1.49 & 27.42 & 3.43 & 434.59 \\
\hline \multicolumn{14}{|c|}{ Cost of Administration-Specific Drug Information Activities } \\
\hline $\begin{array}{l}\text { Planning of clinical pharmacy } \\
\text { services }\end{array}$ & 24 & 19 & 43 & 112.5 & 5.92 & 21 & 21 & 42 & 35.75 & 1.70 & 10.08 & 1.26 & 159.77 \\
\hline Writing statistical report & 14 & 30 & 44 & 317.5 & 10.58 & 14 & 29 & 43 & 48.58 & 1.68 & 17.73 & 2.22 & 281.00 \\
\hline Policies and Procedure design & 9 & 35 & 44 & 300 & 8.57 & 12 & 31 & 43 & 67.46 & 2.18 & 18.65 & 2.33 & 295.64 \\
\hline $\begin{array}{l}\text { Filing of forms/ preparation of } \\
\text { minutes }\end{array}$ & 11 & 32 & 43 & 245 & 7.66 & 14 & 29 & 43 & 50.67 & 1.75 & 13.38 & 1.67 & 212.03 \\
\hline
\end{tabular}




\begin{tabular}{|c|c|c|c|c|c|c|c|c|c|c|c|c|c|}
\hline \multirow[b]{2}{*}{ Type of activities } & \multicolumn{5}{|c|}{$\begin{array}{l}\text { frequent are services provided by } \\
\text { Drug Information Services monthly }\end{array}$} & \multicolumn{6}{|c|}{$\begin{array}{l}\text { time consumed for each service } \\
\text { provided by Drug Information } \\
\text { center monthly }\end{array}$} & \multirow[b]{2}{*}{ 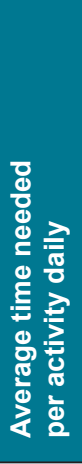 } & \multirow[b]{2}{*}{ 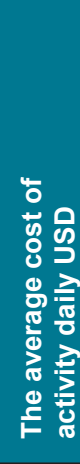 } \\
\hline & 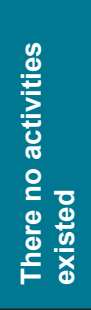 & 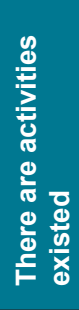 & 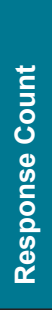 & 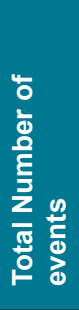 & 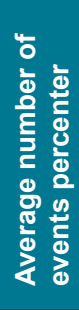 & 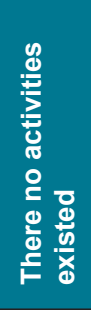 & 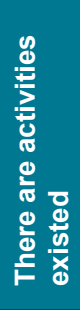 & 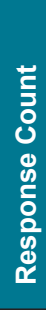 & 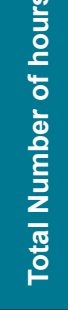 & 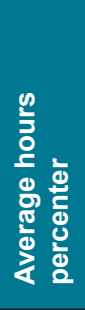 & 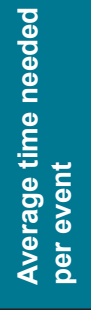 & & \\
\hline $\begin{array}{l}\text { Attending Lectures/ Courses/ } \\
\text { Symposium/ Training }\end{array}$ & 10 & 33 & 43 & 307.5 & 9.32 & 11 & 33 & 44 & 69.67 & 2.11 & 19.67 & 2.46 & 311.81 \\
\hline $\begin{array}{l}\text { Helping in Drug Cases from Outside } \\
\text { Formulary system }\end{array}$ & 17 & 26 & 43 & 150 & 5.77 & 17 & 25 & 42 & 30.96 & 1.24 & 7.14 & 0.89 & 113.24 \\
\hline $\begin{array}{l}\text { Appointments with Pharmaceutical } \\
\text { Co. representatives }\end{array}$ & 20 & 23 & 43 & 127.5 & 5.54 & 21 & 21 & 42 & 22.71 & 1.08 & 5.99 & 0.75 & 95.02 \\
\hline Supervising DIC Services & 14 & 29 & 43 & 282.5 & 9.74 & 8 & 35 & 43 & 63 & 1.80 & 17.53 & 2.19 & 277.92 \\
\hline $\begin{array}{l}\text { Participate on Pharmacy and } \\
\text { Therapeutic Committee }\end{array}$ & 10 & 36 & 46 & 250 & 6.94 & 7 & 36 & 43 & 43.71 & 1.21 & 8.43 & 1.05 & 133.64 \\
\hline Develop update Hospital Formulary & 13 & 31 & 44 & 165 & 5.32 & 8 & 34 & 42 & 75.75 & 2.23 & 11.86 & 1.48 & 187.96 \\
\hline $\begin{array}{l}\text { Setting and evaluating therapeutic } \\
\text { guidelines }\end{array}$ & 13 & 31 & 44 & 192.5 & 6.21 & 14 & 29 & 43 & 62.63 & 2.16 & 13.41 & 1.68 & 212.56 \\
\hline
\end{tabular}

\section{Table 6: The cost of shared drug information center in the hospital committees.}

The frequency of the drug information center participate in relevant hospital committees annually as following

\section{Type of committees}

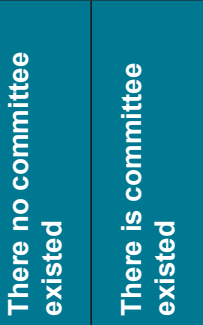

\section{Time consumed for each committee}

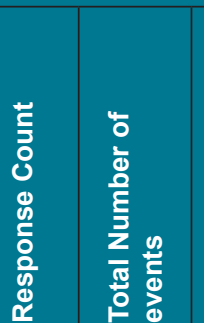

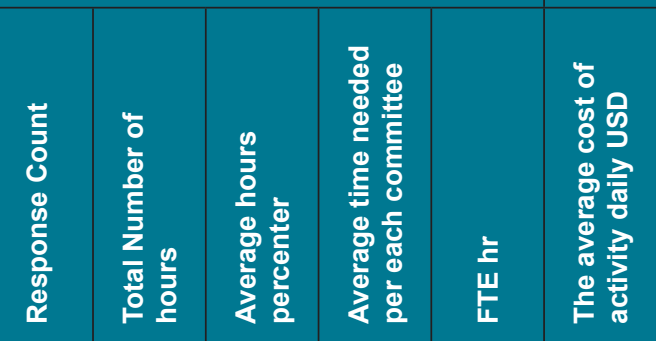

\begin{tabular}{|c|c|c|c|c|c|c|c|c|c|c|c|}
\hline Pharmacy and Therapeutic Committee & 7 & 38 & 45 & 152 & 4.00 & 44 & 42.25 & 0.96 & 3.84 & 0.48 & 60.86 \\
\hline Antibiotics Committee & 15 & 28 & 43 & 78 & 2.79 & 38 & 28.5 & 0.75 & 2.09 & 0.26 & 33.12 \\
\hline DUE Committee & 32 & 10 & 42 & 33 & 3.30 & 35 & 21.25 & 0.61 & 2.01 & 0.25 & 31.91 \\
\hline Infection Control Committee & 24 & 18 & 42 & 74 & 4.11 & 34 & 20 & 0.59 & 2.43 & 0.30 & 38.45 \\
\hline CPR Committee & 27 & 16 & 43 & 55 & 3.44 & 36 & 23.5 & 0.65 & 2.23 & 0.28 & 35.41 \\
\hline Morbidity and Mortality Committee & 34 & 8 & 42 & 29 & 3.63 & 31 & 13.25 & 0.43 & 1.56 & 0.19 & 24.71 \\
\hline Patient or Medication Safety Committee & 13 & 31 & 44 & 78 & 2.52 & 38 & 30.75 & 0.81 & 2.04 & 0.25 & 32.30 \\
\hline Pharmacy and Nursing Committee & 23 & 20 & 43 & 62 & 3.10 & 36 & 26.75 & 0.74 & 2.29 & 0.29 & 36.36 \\
\hline Quality Management Committee & 22 & 23 & 45 & 97 & 4.22 & 37 & 29 & 0.78 & 3.29 & 0.41 & 52.14 \\
\hline Pain Management Committee & 29 & 13 & 42 & 39 & 3.00 & 33 & 18.5 & 0.56 & 1.68 & 0.21 & 26.63 \\
\hline Anticoagulation Committee & 31 & 10 & 41 & 25 & 2.50 & 34 & 18.25 & 0.54 & 1.35 & 0.17 & 21.40 \\
\hline IV Therapy Committee & 32 & 10 & 42 & 25 & 2.50 & 34 & 15.5 & 0.46 & 1.15 & 0.14 & 18.23 \\
\hline
\end{tabular}


(Table 4). The total daily cost of drug information activities was 6,002.18 USD. The highest cost of drug information activities was for central drug information activities $(2,654.69$ USD (44.23\%)) followed by the administration of drug information activities $(2,280.59$ USD (37.99\%)) and patient-specific drug information activities (1,066.9 USD (17.77\%)). The highest daily cost of activities at the central drug information center was toward responding to drug information inquiries (536.24 USD) followed by residential training (274.46 USD) and pharmacist training (263.14 USD). With respect to the administration of drug information activities, the highest daily cost was recorded toward attending lectures, courses and symposiums (311.24 USD), policy and procedures (295.64 USD) followed by writing statistical reports (281 USD) and supervising drug information activities (277.92 USD). The patientspecific drug information activity with the highest daily cost was recorded toward patient counseling (434.59 USD) followed by detection and prevention of medication errors (207.73 USD) and ambulatory care clinic activities (106.94 USD) (Table 5). The highest daily cost of participation of drug information centers in the committee per day was pharmacy and therapeutics committee (60.86 USD) followed by quality management committee (52.14 USD) and infection control committee (38.45 USD) (Table 6).

\section{DISCUSSION}

The drug information services are required as a part of the pharmacy services based on national or international standards. ${ }^{[10]}$ These services can be provided by establishing drug information centers or outsourcing the services through specialized centers. However, outsourcing of drug information services requires that the cost of activities and time needed to perform them are already known. In addition, if the hospital's pharmacy wishes to deliver the services to other organizations, then they need to know the cost of drug information activities. Therefore, we explored the cost analysis of drug information activities at the Ministry of Health hospitals in the Kingdom of Saudi Arabia. The findings of the study showed the total cost of drug information daily activities was high with most of the cost related of the central drug of information activities because most of the time spent on that is one. Followed by administration drug information services less time spent on that activity and patient-specific services because the lowest time spent with pharmacy staff. Among the central activities, the answering of drug information inquiries the highest cost and representative highest time spent while all training section more cost. However, the individual separated training program between residency and pharmacy student. That is a common finding because the highest time and workforce required. In the administration section, the attending symposium the highest cost related to time spent while in the patient-specific the patient counseling was the highest cost due same previous reason. The cost of pharmacy and a therapeutic committee was the highest cost and it was normal because more time in the committee with more activities needed. Followed by quality management and infection control with the highest time needed and following high-cost related activities. The previous findings are difficult to compare with other investigation because of the lacking of information data nationally or internationally. All previous activities are first time calculated and it very useful for outsourced contact or privatizations and fit with new Saudi vision 2030 and Ministry of Health plan in the Kingdom of Saudi Arabia. ${ }^{[11-12]}$

\section{CONCLUSION}

The cost analysis of drug information centers activities is essential to the calculated cost efficiency of drug information services. The common and administration drug information centers services activities are the highest cost. The providing services to other hospitals as outsourced fill with the new vision of Ministry of Health strategic plan in the Kingdom of Saudi Arabia.

\section{ACKNOWLEDGEMENT}

None.

\section{CONFLICT OF INTEREST}

None.

\section{ABBREVIATIONS}

MOH: Ministry of Health; ACCP: American College of Clinical Pharmacy; DICs: Drug information centers; KSA: Kingdom of Saudi Arabia.

\section{ORCID ID}

Yousef Ahmed Alomi (iD https://orcid.org/0000-0003$1381-628 \mathrm{X}$

\section{REFERENCES}


1. Ghaibi S, Ipema H GM. ASHP Guideline on The Pharmacist's Role in Providing Drug Information. Am J Heal Pharm. 2015;72(7):573-7.

2. Alomi YA, Almudaiheem HY, Alarnous T, Alshurei S, Alsharafa A, Alzahrani $\mathrm{T}$, et al. Cost-Efficiency of National Drug Information Center through Ministry of Health Hotline Calling Services (937) in Saudi Arabia: Application of American Model. Value Heal. 2015;18(7):A735.

3. Skoutakis VA, Wojciechowski NJ, Carter CA, Hayes JM, Hudson BL, Martin JA. Drug Information Center network: need, effectiveness and cost justification. Drug Intell Clin Pharm. 1987;21(1):49-56.

4. Lyrvall H, Nordin C, Jonsson E, Alvan G, Öhman B. Potential savings of consulting a drug information center. Annals of Pharmacotherapy. 1993;27(12):1540.

5. Kinky DE, Erush SC, Laskin MS, Gibson GA. Economic impact of a drug information service. Ann Pharmacother. 1999;33(1):11-6.

6. Marrone CM, Heck AM. Impact of a Drug Information Service: Practitioner Hours Saved. Hosp Pharm. 2000;35(10):1065-70.

7. Ponampalam R, Loh CS. Cost benefits of the Drug and Poison Information
Centre in preventing unnecessary hospitalization: The Singapore experience. Hong Kong J Emerg Med. 2010;17(1):45-53.

8. Brown JN. Cost Savings Associated With a Dedicated Drug Information Service in an Academic Medical Center. Hosp Pharm. 2011;46(9):680-4.

9. Bond CA, Raehl CL, Franke T. Clinical pharmacy services, pharmacy staffing and the total cost of care in United States hospitals. Pharmacotherapy. 2000;20(6):609-21.

10. Alomi YA, Mudaiheem HA. National Drug Information Center Services through Ministry of Health Hotline Calling Center (937) in Saudi Arabia. Adv Pharmacoepidemiol Drug Saf. 2016;5(1):1-7.

11. Government of Saudi Arabia. Saudi Arabia Vision 2030. 2016. Available from: https://vision2030.gov.sa/sites/default/files/report/Saudi_ Vision2030_EN_2017.pdf

12. Alomi YA. New Pharmacy Model for Vision 2030 in Saudi Arabia. J Pharm Pract Community Med. 2017;3(3).

Cite this article as: Alomi YA, Aldosary BA. Cost Analysis of Activities for Network Drug Information Centers at the Ministry of Health Hospitals in Saudi Arabia. Int J Pharmacol. Clin. Sci. 2019;8(1):45-51. 